\title{
Polarized Light Microscopy
}

\author{
Dragana Rančić
}

\begin{abstract}
Polarized microscopy and a variant of it polarized Raman spectroscopy are techniques that are used for analysis of anisotropic objects. It gives information on absorption, colour, structure, composition and other characteristics of different substances thus affording characterization and identification of variety of biological materials.
\end{abstract}

\section{Polarized light microscopy}

Polarized microscopy includes ilumination of the sample by polarized light. Normal, unpolarized, light (originated from both natural sunlight or most forms of artificial illumination) can be thought of as many sine waves, each oscillating at any one of an infinite number of planes around the central axis, while polarized light oscillates only in one plane. Unpolarized light can be transformed into polarized light, passing throught the special filter permitting the passage of light oscilating in only one plane (Fig. 1) (Collett 1993).

How to cite this book chapter:

Rančić, D. 2019. Polarized Light Microscopy. In: Vucelić Radović, B., Lazić, D. and

Nikšić, M. (eds.) Application of Molecular Methods and Raman Microscopy/

Spectroscopy in Agricultural Sciences and Food Technology, Pp. 193-198. London:

Ubiquity Press. DOI: https://doi.org/10.5334/bbj.p. License: CC-BY 4.0 


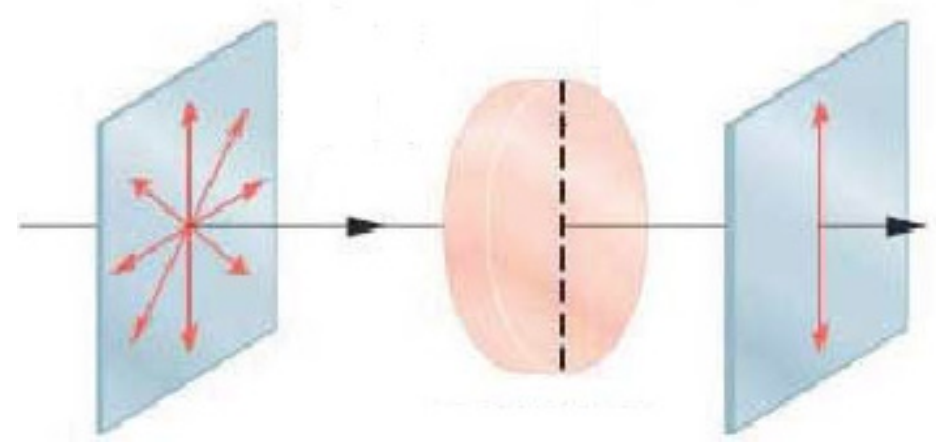

Figure 1: Polarizing filter allows passing only the light waves vibrating in a specific direction.

Microscopic examination using polarized light is especially useful for investigations of anisotropic objects. Isotropic materials, like unstressed glasses and cubic crystals, demonstrate the same optical properties in all directions, while anisotropic materials have optical properties that vary with the orientation of incident light. Around $90 \%$ of all solid substances are anisotropic. When these anisotropic materials are rotated, the observer may see brightness and color changes under polarized light that depend on the orientation of the material in the light path. This technique allows researchers to obtain information on color absorption, structure, composition, light refraction and other properties of different substances what can be used to characterize and identify various materials. Specimens having more than one index of refraction (eg. many organic as well as inorganic crystalline materials) produce birefringence effects, meaning that in field of view bright against the black background will appear. Views of some biological samples in polarization light can be similar to the darkfield technique, but polarized light is a contrast-enhancing technique that improves the quality of the image obtained with birefringent materials.

The polarized light microscope is equipped with two polarizing filters: first one (called polarizer) is positioned in the light path somewhere before the specimen, and the second one (called analyzer) is placed in the optical pathway after the objective rear aperture (Collett 1993). Polarized light is created by passing light through a polarizing filter, which transmits light in one direction only. An analyzer determines the amount and direction of light that illuminates a sample and image contrast arises from the interaction of polarized light with a birefringent specimen. When both the analyzer and polarizer are in the optical path, their permitted vibration directions are positioned at right angles to each other, so no light passing through the system and a dark field of view present in the eyepieces. Therefore, the image that is projected by the objective 

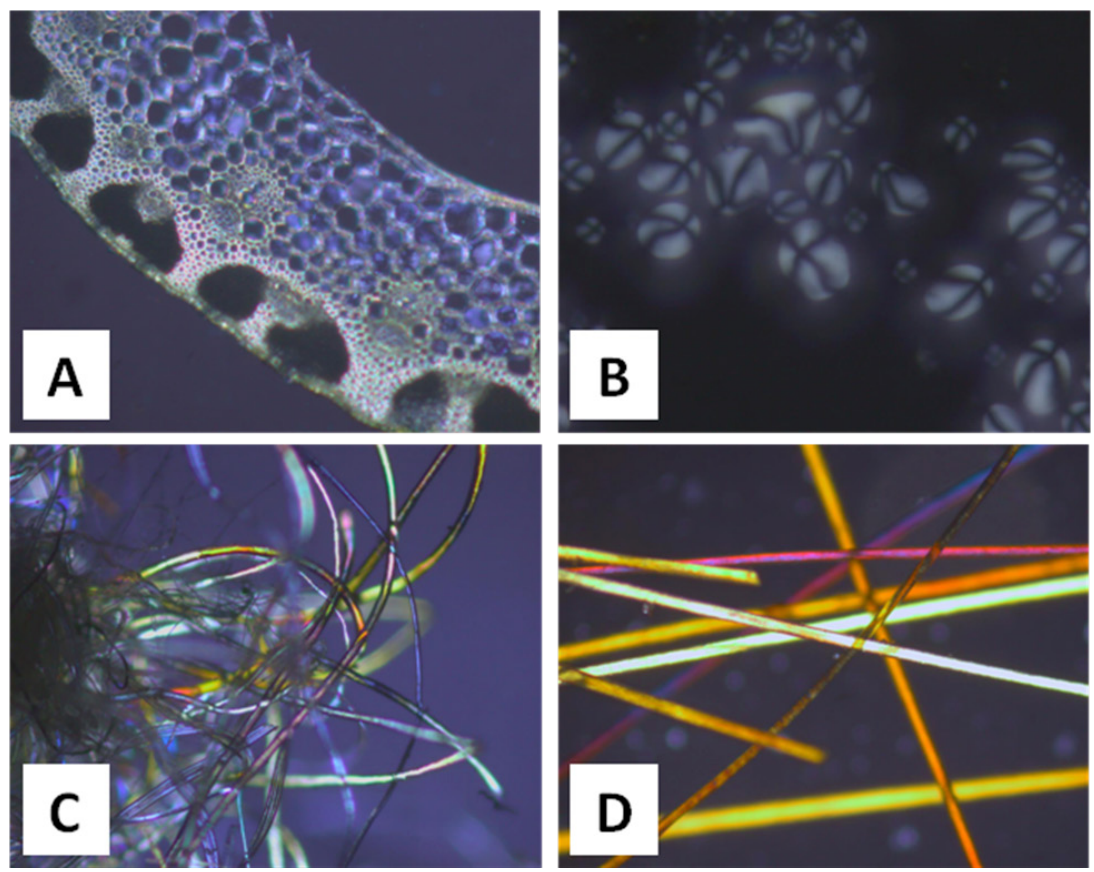

Figure 2: Some biological samples observed in polarizing light microscope: A- wheat stem cross section, B- potato starch, C- wool, D- human hair.

is mostly dark, except for bright specimen parts that are bierefringent or otherwise optically anisotropic (Fig 2).

Polarizing microscopy can be used with both, reflected or transmitted light. Transmitted light refers to the light diffused from below the specimen. This light is often passed through a condenser, which allows the viewer to see an enlarged contrasted image. Reflected light is especially useful for the study of opaque materials, such as metals, alloys, mineral oxides and sulfides.

Polarized light microscopy has a wide range of applications. The technique can be used both qualitatively and quantitatively in materials science, geology, chemistry, biology, metallurgy and medicine. Although polarized microscope is perhaps best known for its geological applications and is primarily used for the study of rocks and minerals, it can also be used to study many other materials including both natural and industrial minerals, composites such as cements, ceramics, mineral fibres, polymers, and crystalline. It is also useful in forensic examination. The various components in several biological specimens display beautifully in polarized light: different plant parts (Fig. 2A) especially fibres and wood, trichomes, pollen and starch grains (Fig. 2B) (Henry et al. 2011), bones, fish scales, wool (Fig. 2C) and hairs (Fig. 2D). It is also possible to observe cell 
division process and cell arrangement in tissues (Ivanov \& Ignatov 2013), cellulose microfibrils and other details of plant cell wall (Leney 1981), silica body in plants (Dayanandan et al. 1983), numerous opaque and/or "thick" specimens and a wide variety of other specimens.

\section{Polarized Raman scattering}

Polarized Raman spectroscopy is a vibrational spectroscopic technique where the polarization of light which has been subjected to Raman scattering by a sample is determined. Raman spectroscopy is an ideal technique for polarization measurements because the lasers used for excitation emitte linearly polarized monochromatic light. But since Raman results can be misleading if one does not account for laser polarization, in conventional general purpose instruments the laser excitation beam is intentionally depolarized so as to give consistent results regardless of sample orientation. Advanced Raman instruments can be configured to provide, in addition to conventional Raman measurements, also polarized Raman measurements. In polarized Raman spectroscopy the polarized Raman scattering is observed as a result of the interference of the polarized light with the molecules vibrating. By using Raman polarization analyzer, the precise directional information about the differential polarizability of the molecules can be obtained, so polarized Raman spectroscopy probes provide insightful information such as molecular orientation and symmetries of the bond vibrations. Therefore, in addition to the general chemical identification which conventional Raman provides, these technique can improve the quality and quantity of the hyperspectral Raman dataset (Chiu et al. 2015) and it helps to reduce the depolarized auto-fluorescence backgrounds over the polarized Raman bands (Thomas et al. 1995).

This technique has been developed and usually applied for the chemical and physical analyses in material since, mainly in the aim to provide useful information relating to molecular composition and orientation in synthetic and natural polymer systems (Jarvis et al. 1980, Ward 1985, Bower 1972), but recently the role of polarization-resolved Raman spectroscopy in life science increased, opening new possibilities in monitoring biochemical composition, structures and symmetry of vibrations in biological tissues. It has already been used in the studying of various biomolecules including proteins, nucleic acids, crystals and fibers (Fanconi et al. 1969, Tsuboi et al. 1996, Tsuboi et al. 1997, Ko et al. 2006, Lim et al. 2011), in determining detailed architecture of viruses (George and Thomas 1999), evaluating three-dimensional collagen fibril orientation in tissues (Galvis et al 2013), Benevides et al 1997) or obtaining more chemical information from living cells (Chiu et al 2015). This technique also has applications in pharmaceutical science (Kiefer 2017) and has good diagnostic potential in medicine, for example in detection of cancer cells (Pachaiappan et al. 2017, Abramczyk et al. 2018). 


\section{References}

Abramczyk, H., Brozek-Pluska, B., Kopec, M. (2018). Polarized Raman microscopy imaging: Capabilities and challenges for cancer research. Journal of Molecular Liquids, 259: 102-111

Benevides, J.M., Tsuboi, T., Bamford, J.H.K., Thomas, G.J. Jr. (1997). Polarized Raman spectroscopy of double-stranded RNA from bacteriophage $\varphi 6$ : local Raman tensors of base and backbone vibrations. Biophysical Journal, 72:2748-62

Bower, D. I. (1972). Investigation of molecular orientation distributions by polarized Raman scattering and polarized fluorescence. The Journal of Polymer Science (Polym. Phys.), 10: 2135-2153

Chiu, L, Palonpon, A. F., Smith, N.I., Kawata, S., Sodeoka, M., Fujita, K. (2015). Dual-polarization Raman spectral imaging to extract overlapping molecular fingerprints of living cells. Journal of Biophotonics, 8, 546-554.

Collett, E., (1993). Polarized Light: Fundamentals and Applications. Marcel Dekker, New York. ISBN-13: 978-0824787295

Dayanandan, P., Kaufman, P. B., \& Franklin, C. I. (1983). Detection of Silica in Plants. American Journal of Botany, 70, 1079-1084.

Fanconi, B., Tomlinson, B., Nafie, L.A., Small, W., Peticolas, W.L. (1969). Polarized laser Raman studies of biological polymers. The Journal of Chemical Physics, 51(9):3993-4005.

Galvis, L., Dunlop, J.W.C., Duda, G., Fratzl, P., Masic, A. (2013) Polarized Raman Anisotropic Response of Collagen in Tendon: Towards 3D Orientation Mapping of Collagen in Tissues. PLoS ONE 8(5): e63518.

George, J., Thomas, Jr. (1999). Raman Spectroscopy of Protein and Nucleic Acid Assemblies. Annual Review of Biophysics and Biomolecular Structure, 28:1, 1-27

Henry, A. G., Brooks, A.S. , Piperno, D. R. (2011). Microfossils in calculus demonstrate consumption of plants and cooked foods in Neanderthal diets ((Shanidar III, Iraq; Spy I and II, Belgium). Proceedings of the National Academy of Science of the United States of America, 108, 486-491. DOI10.1073/pnas. 1016868108

Ivanov, O. V. \& Ignatov, M. S. (2013). 2D Digitization of Plant Cell Areolation by Polarized Light Microscopy. Cell and Tissue Biology, 7, 103-112.

Jarvis, D. A,. Hutchinson, I. J., Bower, D.I. and Ward, I. M. (1980). Characterization of biaxial orientation in poly(ethylene terephthalate) by means of refractive index measurements and Raman and infrared spectroscopies. Polymer, 21: 41-54.

Kiefer, J. (2017). Polarization-Resolved Raman Spectroscopy for Pharmaceutical Applications. American Pharmaceutical Review.

Ko, A. C.-T., Choo-Smith, L.-P., Hewko, M., Sowa, M. G., Dong, C. C. S., and Cleghorn, B. (2006). Optics Express Research, 14 (1): 203-215 
Leney, L. (1981). A Technique for Measuring Fibril Angle Using Polarized Light, ISSN : 0043-7654

Lim, N. S. J., Hamed, Z., Yeow, C. H., Chan, C., and Huang, Z. (2011). The Journal of Biomedical Optics, 16(1): 017003.

Pachaiappan, R.; Prakasarao, A.; Singaravelu, G. (2017). Polarized Raman spectroscopic characterization of normal and oral cancer blood plasma. 100541F. 10.1117/12.2255600.

Thomas, G. J. Jr., Benevides, J. M. , Overman, S.A., Ueda, T., Ushizawa, K., Saitoh, M,Tsuboi, M. (1995). Polarized Raman spectra of oriented fibers of A DNA and B DNA: anisotropic and isotropic local Raman tensors of base and backbone vibrations. Biophysical journal, 68(3): 1073-1088.

Tsuboi, M, Overman, S.A., Thomas, G.J. Jr. (1996). Orientation of tryptophan 26 in coat protein subunits of the filamentous virus Ff by polarized Raman microspectroscopy. Biochemistry 35:10403-10

Tsuboi, M., Thomas, G.J. Jr. (1997). Raman scattering tensors in biological molecules and their assemblies. Applied Spectroscopy Reviews, 32:263-99

Ward, I. M. (1985). Determination of molecular orientation by spectroscopic techniques. In: Kaush H.H., Zachman H.G. (eds) Characterization of Polymers in the Solid State I: Part A: NMR and Other Spectroscopic Methods Part B: Mechanical Methods. Advances in Polymer Science, vol 66. Springer, Berlin, Heidelberg. ISBN 978-3-540-13779-5 